\title{
Homo Digitalis - in Search of a Patterned Usage Identity
}

\author{
Adrian STOICESCU \\ University of Bucharest, Romania \\ adrian.stoicescu@litere.unibuc.ro
}

\begin{abstract}
There is a great debate around the ways identity is shaped online, mainly as a result of understanding the online as networked individuals. The present papers tries to open the discussion on whether the quasi-synonym words constantly used to talk about the online communication cyber, virtual and digital define the same reality or whether they can be associated with particular aspects of the identity forged within the computer mediated communication. The search for the differentiating aspects is carried out against the backdrop of technological development which inevitably alters with every new aspect unveiled a new side of each individual's identity.
\end{abstract}

Keywords: Identity, Cyber, Virtual, Digital, Historical Development

1 Introduction

More than four decades ago, Clifford Geertz [1] began the analysis of his The Interpretation of Cultures by referring to Susanne Langer's views on great ideas which, as she understood, appear all of a sudden to offer a solution to all the questions unanswered before or to shed a new light on those that had already been answered. Recontextualisation appears to be a sort of a key in reading the world as it has never been done before. But the overuse of such terminology and the suitable-in-all-fields feature it may hold for a time eventually backfires. Such great ideas alongside their great wordings wear out becoming nothing more than buzz words. Strictly speaking, as Geertz suggests, the great ideas only transiently suffice the multitude of fields they may comprise of on various degrees.

On the other hand, before proving their worthlessness and passing into the oblivion of the scientific discourse, such concepts become the primary focus of epistemological debate under constant struggles to enlarge and to fine tune their meaning. Far and away, the greater the complexity of the era is, the bigger the number of concepts defining the way the scholars narrate about their surrounding realities. Today, such concepts are, among others, memory, trauma and identity emerging as intrinsic to all social sciences discourses and combining in various ways to become sufficient in depicting the constantly changing cultural realities. The large array of fields which incorporate in their texts such concepts on the other hand erode their capacity of deciphering realities and the constant scientific hype around them might eventually render those useless. A fortunate way of escaping from a conceptual uselessness lays within the use of determiners, functioning both to delineate the filed in which they are used and to grade various particular usages within a certain field.

With an ever increasing pace of development and ubiquitous presence, the field of computer-mediated communication makes no exception. The scientific discourse constantly borrows from adjacent narratives of social science leading thus to a networked conceptual frame pretty much in the sense of the networked individual it seeks to analyse. This is, in fact, the purpose of this article: to try to define a type of identity stemming from the contemporary use of technology, the digital identity.

\section{Identity}

Incorporated in the anthropological discourse of the $60 \mathrm{~s}$ and $70 \mathrm{~s}$ of the $20^{\text {th }}$ century, the concept of identity migrated from one discipline to another and changed its meaning quite a few times [2], [3]. Of the multitude of definitions and perspectives the concept incorporated over the years, only 
those useful to this paper will be reviewed here.

Generally speaking, the central meaning today, resulting from the binary opposition with otherness, could be narrowed down to an intricate exercise of self-identification with a group or the self-identification of a group, whereas such identification is, in fact, a constant cultural negotiation for achieving various degrees of sameness or, on the contrary, paradoxically, various degrees of difference. Identity is so seen as a process of constructing (rather than the construction itself) based on independent (or individualistic) and interdependent (or collectivistic) cultural construal's` [4]. The fear of essentialism leads the discourse away from a type of reification it was subjected to thus enabling identity to become a synonym for multiplicity, fluidity and fragmentation [5].

The trinomial enlargement of meaning may be seen as a direct consequence of the fact that the negotiation of identity is performed between the individual characteristics, i.e. personality traits, values and preferences, etc., and the social one, i.e. the roles a person plays in the group [6].

But, it is crucially important to mention that the individual vs. the social characteristics in shaping identity is hardly an invention of recent trends in conceptual appropriation, the uneven weigh they carry having shifted repeatedly over time. One of the classics of identity studies, Erving Goffman, in his ground breaking The Presentation of Self in Everyday Life deems identity as perfomative, picturing it as theatrical and understanding it in its mobility of the role. For him, performance represents: 'all activity of an individual which occurs during a period marked by his continuous presence before a particular set of observers and which has some influence on the observers (...) [and this performance] regularly functions in a general and fixed fashion to define the situation for those who observe the performance' [7].

By sheer contrast, although both meanings of identity presented above capitalize in the same interactions manner on the relation between the individual and the group, performativity alters dramatically from the emphasis placed on the group, in the early work of Goffman, to that on the individual, as identity is represented now. A multiple, fluid and fragmented identity is to be found on both definition leading to the idea of a very strong connection to the context of performing. But while for Goffman the group shapes the individual performance with a view to select from the multiple, fluid and fragmented identities a person has in order to achieve sameness to any given circumstance, Butler's identity pictures the group as the reality against which the individuals may act or, most importantly, react, and enact various aspect of the individual identity facets, for purposes of differentiation.

Goffman's perfomative identity represents a kind of being tailored to the group expectations in a sense of conformity, while Butler's is a kind of doing [8] exploring the individualities in a solid grasp of conflicting disagreement.

Furthermore, these two definitions picture perfomative identity first as means of empowering individual traits to fit a specific socio-cultural context, while the second converts these to a sort of denial, the appropriation of the context traits being made to be tailored to the individual.

\section{Cyber, Virtual, Digital as Online Identities?}

Narrowing down the concept of identity to the software-dependant environment, it comes natural to say that the fluidity of identity is mainly determined by constant transformation the technology is subjected to. Each and every hardware or software upgrade immediately reverberates through the intricate process of identity building.

To begin with, the matters related to identity building within the larger context of the human computer-interaction processes must be clarified in the sense that, although much of the studies condense over the computer mediated-communication, that intrinsically contain the online component, there is 
another smaller but far from being neglected aspect related to this interaction in connection free contexts. It is here the place where one should consider the early stages of that particular interaction with the devices that run on a particular software that does not require connection to the Internet.

Surprisingly or not, they were designed for children in the form of animated toys and offered the first glimpse of "computers in the culture of living things' [9]. These toys offer, as it shall later on be seen, a strong identity component to the first stages of possible classification in the identity shaping process. The second type of identity construct on the realm of human-computer interaction is the special type of individual or group identity building process in the online environment which is polarised around connection. Irrespective of the stage of its technological development, but in a reductionist view, the rather simple Internet architecture consists of individual pages all linked together. It pretty much doubles the way the humankind exists as a network, but on a virtually infinitely higher scale. In fact, O'Neil defines the Internet in such words as 'a network, a connection of nodes by ties. Any node on the Internet is accessible from any other node, and there are no differences between the ties that connect the nodes: all hyperlinks are equal' [10] which, in turn, enables a 'spontaneous expression and organisation [11].

But such a perspective on the Internet is both self-contained and rather useless for the user generated content perspective that moves away from the Internet as an object to the Internet as a medium. Additionally, for the purpose of placing under scrutiny the onliners' identity, the Internet definition leaves aside the technical devices that function as gateways to it. In other words, not only the Internet as either object or medium is relevant, but the computer (irrespective of its shape) too, especially if the identity building process espouses the historical perspective.

Having this in mind, the question to be asked that stems from this periodization is to what extent the words cyber, virtual and digital can actually represent individual types of identity or rather patterns of exclusion relevant for the development of interaction, and, at the same time, being the hyponyms of the types of interaction with or enabled by a softwared device. Of course, this association does not represent a definitive way of classification but rather an opening path to further discussion mainly due to the fact that they are interchangeably used in the online research fields today.

Furthermore, the three words are very rarely used as nouns but rather as adjectives (or determiners) collocating with basically every word used for the scientific approach to the software-animated devices: cyberspace, cyberpl@y or cyber greeting [12], virtual worlds, virtual relationships, virtual identity or communication, virtual reality, virtual persona and the list may go on. Even the disciplines themselves negotiate the terminological use, oscillating between virtual / digital ethnography or anthropology or, furthermore, with rather limited usage, cyber-ethnography [13].

Reading thus the recent text on various aspects related to the online identity people display might become a conundrum since concepts appear to be used as it may be handy at a certain moment. Only one sample of such randomly picked texts might be enough in order to justify the need for terminology clarifications: 'based on virtual ethnography and discourse analysis of online arenas that are central to Israeli transgender community, the study indicates that transgender users employ cyberspace in three main ways: as preliminary, complementary, and/or alternative spheres. By paying close attention to cyberspace as an alternative sphere, which is especially pertinent to the transgender users, the paper revisits two central issues in Internet research, namely the relationships between the online and the offline worlds, and identity issues (authenticity versus falseness) within the online settings' [14].

Quoting such a long piece of text is not for the purpose of a critical reading of the 
author's undertaking, but for that of seeing how the language of this constantly developing scientific field may be insufficient or confusing when describing its object. In term of scientific approach, the author states his methodology as belonging to the field of virtual ethnography by means of which he intends to conduct a research on the Internet, embracing together both the online and the offline, by conducting participant observation and discourse analysis in the cyberspace. What he basically does here is to put together virtually all the words at hand at the moment which, even though can be synonyms to a certain point, as we shall from this point later see, are but synonyms.

The first aspects to be contextualized revolve around cyber which, when used in the field of human computer interactions, statistically speaking, takes the form of the compound nouns cyberspace. The first use of the term appears on the other hand to have more to do with the fiction rather than science, the person being credited with the creation of the term being William Gibson in his 1984 novel Neuromancer [15] in which the main character displaces himself on a realm of the networked computers where he finds 'an alternative and rather dark social universe whose interaction with real life drove the novel's plot'[16].

Although besides creating the word nothing else appears to be standing out of Gibson's word the escape behind the computer network reality may offer a clue on potential meanings of this word. The matter of alternative reality is what one should be reminded of when it comes to this, and, more importantly, that of navigability [17]

Strictly lexically speaking, cyberspace belongs to rather significant in number family of words, parented as it appears in its present meaning by the 1948 Norbert Weinder's term, cybernetics. Cognate among themselves by the compound element cyber, the list is varied and complex: cyberpunk, cyberculture, cyberlife, cybernauts, cyberselves, cybersec cybersociety, cybertime [18], to which a great deal of other words might me added including the cyborg, this last one carrying a certain degree of additional meaning for the subsequent undertaking in the discrimination among meanings process.

Besides a comprehensive review of the definitions cyberspace benefited from during its short but intense scientific depiction life, Strate conceptualized it in the form of a complex structure of superimposed layers of physicality, meanings and perception: 'to use an architectural analogy, the zero order is the equivalent of a building's basement or lobby as opposed to the first floor. The next level I refer to as first order cyberspace; it focuses on cyberspace's basic elements or building blocks. The final level is the second order cyberspace; it represents synthesis of those basic elements.' [19]

Intended as a taxonomy of cyberspace, Strate's concept charting [20] is extremely valuable since it combines the referenced sources in a wider definitions that are transparent in the sense of a chronology following each author's approach and, most valuably the connection between the individual and the very diverse degrees of coherency displayed in the relations to the softwared object.

Strate's up to date to the year 1999 taxonomy coalescences idea of space both as strictly fictional (in the traditions inaugurated by Bukatman or Gibson) and relational as with the all other definitions reviewed. Furthermore, the relational feature such definitions share is tightly related to the idea that cyberspace is mind-generated as a result from the human-computer interaction, or quite differently, it is generated by the computer interface and the human senses. Last but not least, cyberspace is defined by the material base of the hardware and the usage they are subjected to.

But on the other hand, such a great agglutination of definitions and conceptual appropriations leaves the impression that cyberspace in a very wide reality combining fact and fiction, materiality and mind projection, relations and individual experience. 
In fact, it is the place here to look back to what human-computer interaction really represents. The present meaning of computer irrevocably links it to the idea of connection to other similar devices, which is in fact the corner stone of the networked-self as it may appear in various discourses. This is the reason why I preferred so far the use of the softwared devices instead of other phrases in order to cover the reality of the first such devices that were not able to connect. From this point of view, the relational nature of cyberspace must be taken into account, which, in fact, leads to three types of distinct relations: the first, between the human and the software device which is unable to connect, the second, the human and the connectable devices, whilst the third is the core of the computer-mediated communication, the relation between humans via a software connectable device. It is my belief that this tri-level relation classification can on the other hand offer a key in reassessing the history of concepts in the field of human-computer interactions.

At the same time, one should also take into account another factor of discontinuity when it comes to defining the cyberspace and identity: the digital natives [21]. Identified in connection to the education studies, the digital natives oppose the digital immigrants as being brought up in a computer world opposes the learning process of a second language.

Sherry Turkle [22] opens her ground breaking 1984 study by addressing issues surrounding the novelty represented by the rather rudimentary devices that run on even a more rudimentary software which children computer toys represented back then, followed by the more complex reality of video games, both in arcade and at home, or the early impact of introducing computers in schools. Either questioning the human nature of such device or trying to understand those in their own particular system of meaning, the early forms of computers represent, as Turkle pinpoints unarguably, an integral part 'in their process of world and identity construction' [23].
Without any doubt, or at least for the digital natives, computers not only build up identity but they also validate it. The question that springs to mind now is to what extent they exert influence on this process of growing up technologically and, moreover, which is the measurable contribution easily distinguishable in their identity profile. This question, rather difficult to answer this day, appears to have a solution in Turkle's analysis. Irrespective of the complexity of a computer, 'a medical diagnosis, a move in a chess game - all the computer really does is add. [24]'

Whether it betters an old functionality or develops new ones, whether it sharpens perception or opens new mediated ones, this early stage in computing technology is no more or no less than an add on to humans. This early assumption on what the computer does for the humans (and less on what the computers does to the humans), opens the grounds for bringing forth the previously mentioned concept of cyborg.

Although helpful to a certain extent to understand what cyberspace is and additionally how identity takes shape, the concept of cyborg proves to be equally challenging when it comes to being defined. The understanding of a cyborg as a coherent combination between human and mechanical or electronic parts is easily transcended by picturing it as 'all entities that carry human culture' [25], basically capitalising further on what Turkle hinted at as the addition provided by a computer. But these cultural bearing devices are moreover subject to an adaptive nature, pretty much like the natural beings adapting to the environment [26]. To this end, the cyborg is definitely seen as a user responsive entity both in complying with the tasks it receives and in the sense of its very own adaptive nature to the new requirements from its users which are converted in new capabilities the software provides.

The cyborgic adaptive nature of software devices is on the other hand specific only to two of the three categories of relations mentioned earlier, namely human-human 
interaction mediated by the softwared devices and that between the humans and the connectable ones.

The introductions of connectivity to all the discussion here recasts the understanding of the devices and contiguously the process of the identity building they are a part of into a new kind of mould in which the cyborgic nature of space becomes dramatically altered. Overstepping the views picturing the computer as a tool and growing increasingly distant from an exclusive utilitarian understanding of computing, the introduction of the Internet brings a third variable to the binomial relations. Especially synchronous its mass accessibility the identity build online now is pretty much carved out of the bulk of features the Internet has. First, the negated geometry and the death of distance [27] creates in the connected world of the online the 'potential of immediacy and the experienced reality of waiting for information to download' [28].

Of course here Hine literally refers to the actual process of retrieving numbers of bytes of information either in the form of a file or in that of accessing the informational content of a site, and thus consuming the pieces of identity the other, the skilled ones, to paraphrase O'Neil, who will later on, once the web 2.0 is developed, become the authoritarian figures that shatter into pieces the equalitarian structural view on the Internet by incorporating 'structural inequalities' and a sense of domination [29].

But above this, the moment of great importance in marking a total switch in the way the individual becomes involved with the online is that when the content hosted here becomes user generated. I will deliberately jettison matters of identity related to either solitude or togetherness, online / offline continuities or discontinuities, utopian or dystopian orders the online may offer. Although relevant to a great extent and very much debated over in huge series of pros and cons they fall aside the purpose of this present undertaking. Indeed all the binary oppositions above can be easily perceived and form integral parts of computer-mediated performed identities. And equally true such identities are shaped on the realm of fluidity, multiplicity, and fragmentism as stated at the beginning of this paper.

Yet such identity features although unevenly identifiable in all types of online interactions which may be reduced to only two contiguous dimensions - with the content and with the other users - seem to embody realisations on at least two levels of content the cyberspace comprises of. These two levels on the other hand are but dissonant: the cyber and the virtual. The latters is but a portion chiselled out of the much larger environment of cyberspace.

Virtual reality is 'a new kind of environment, created by human perceptions triggered or mediated by a video computer technology' [30]. Pretty much identical to the cyberspace itself, becoming now its synonym for the same mediated reality in which the content can be modified, personalised, incorporated or denied, virtuality is thus only a part of cyberspace. It is that part of literally fictionalised existence benefiting from what Coleridge defines as suspension of disbelief [31] in the immersion in a very particular type of environment. Although constantly associated with literature the very essence of which it is, fiction is embodied on the other hand an as constitutive element of a large portion of the Internet in which it actually shapes the individual identity. This fictional identity located on the realm of cyberspace is, unlike other types of fiction, at the same time performative and conditioned, and exclusively found on web-based platforms like Second Life and pages from Facebook or MySpace [32] to name just a few.

Virtual worlds on the other hand must be defined resorting again to binary oppositions. They oppose the real worlds to which they still remain linked [33] and, most importantly, they oppose a part of cyberspace which paradoxically they are a part of in some constitutive elements which are the recreation of the real world.

The virtual worlds and implicitly the virtual identity oppose the real world in the sense 
that they are a fictional derivatives of it, but, most importantly, they do not constitute and antagonistic facet of humans, nor do they represents a recent addition to the humankind contemporary to the technological boom from the last couple of decades. In the World of Techne, Boellstorff drawing on Turkle's work considers virtuality as an opening of a different platform of the operating system it is programmed to function in the way to incorporate its relation to technology [34].

This world of games, the virtual, can thus be seen pretty much in the complex manner Goffman understood the performed identity as a 'front stage' undertaking. It is on the other hand, a way to further take the idea of performance. The virtual identity first conditionally creates its world, its rules, and its patterns of interaction so that only after it can be literally performed. In fact, such world resemble a lot fairy tale in the sense of its fictional conditionality. A preset system of blocks of action or functions as V.I. Propp [35] names them are selected and combined in order to make the narration of a folktale. Pretty much similar, a virtual world is also combined from a predefined set of images this time, which may be in the form of some sets of user actions the alteration of which is rather less possible. With various degrees of modification possibility these can be combined and tailored to the user's needs so that they eventually incorporate into a coherent patterned interaction that form the virtual life narrative.

But, if in the case of the folktale the functions come from a generally accepted oral community system of values and the incorporation of such functions answers the general views of such community, in the case of the virtual worlds the negotiation process of building the narrative takes part between the users and the virtual world general frame made by the designers who "have certain goals and desires about what people will do' [36]. But if in a folktale the degree of performance is limited to only a few elements the teller might add when narrating, the performance in the virtual worlds is extensively shaped as interactive story telling. Not only does each individual create a fictional virtual world of its own but at the same time it enacts the narration which is actually now 'written'.

To sum it up, the virtual world which generates the virtual identity is only one limited side of the much larger cyberspace. It incorporates literally the creation of a personal narrative which the user both 'writes' and 'plays' the entrance in which following the rules of fictions (be it mimetic or not) in the sense literature allows the creation of new worlds. Furthermore, such worlds and their identities are conditional, in the sense of adjusting the narrative to the technical constraints the designers impose and performative in the sense of simulating the existence.

\section{Homo Digitalis}

The incorporation of the digital in the discourse about the human-computer interaction is a novelty and its attributive use is linked to the creation of a new discipline, the digital anthropology. Claiming 'the profound theoretical silence' [37] surrounding the term digital, Boellstorff dismisses the digital understood as a string of $0 \mathrm{~s}$ and $1 \mathrm{~s}$ and, resorting to the indexicality theory from linguistics, concludes that the digital is the indexical sign for both online and offline. Bearing this in mind, he interprets digital anthropology as being 'more than just a study of things you plug in or even the study of Internet-mediated sociality' [38].

Although very coherent in the sense of determining what digital anthropology is, the author only drops hints at what the digital may be when it does not collocate with anthropology. A by far more useful account to this end is Roger's [39] who, advocating the end of real-virtual divide, again for reasons of methodological clarifications, draws the conclusion that the computer media are not so much relevant for representing refashioned media but more for their capacity of production or, to put it in other words, poetic dimension must be completed by a poietic one. 
The digital identity will become synonym to first, the latest form of the Internet embodying features of content generating processes, second, the consumption of such user-generated content in order to fill in gaps of knowledge, relations, and emotions, and third, the abolishing of the strong sense of discontinuity between the technical and the non-technical sides of the individual.

Keeping these three features in mind, the homo digitalis reveals under at least three facets which cognate to the cyber and virtual as understood before but which nevertheless bear the specificities of the technological development.

Attempt to classify internet users are a rarity in the anthropological discourse since the focus was almost exclusively on niches of how human behave on the online environment. Not surprisingly, such classification come from the marketing specialists who, in order to target better their activity, were interested in the patterns of Internet usage.

One such attempt is Kozinets' who, integrating the individual in the online community (and not communities understood as theme driven associations) by means of the degree of involvement in the online, identifies four types of users: the newbies, the minglers, the insiders and the devotees [40]. Furthermore, Kozinets identifies at the same time some patterns of interaction based on the intensity of the relations, i.e. geeking, building, bonding and cruising [41]

On the other hand, another study available online, conducted on 2000 British consumers by Davin Zeitlyn who claims he authored the 'fist digital anthropology report' [42] but who might only benefit from the novelty of his title collocation, and posted by Jukka Jouhki, takes such research a step further. The six tribal categories of homo digitalis the author identifies benefit from an in depth identification in terms of gender, age, active profession and income weighed against the British average. These are the digital extroverts, the timid technophobes, the social secretaries, first lifers, e-ager beavers, and, last, web boomer.
Of course the rather ludic way of presenting his categories questions the scientific value of such findings, but leaving aside the not so very fortunate way of labelling some of the categories, one can still perceive the rather strong social discontinuities the Internet users represent when it comes to extrinsic factors of classification. This does not lead to the idea that uniformity is what the study of the homo digitalis seeks, nor that such patterns will try to categorise extensively how people will shape identities by dissecting the patterns of interaction to their smallest bits.

The identity of homo digitalis will take shape here by observing two types of interactions, that with the informational content and that with the other users of the digital environment. The first category of observation includes both the producing and the consumption of web content, irrespective of its types, ranging from 'utilitarian' information (banking, schedules, online buying, research on various aspect of everyday life etc.) to 'non-utilitarian' one (gaming, Facebook or Tweeter post, etc.), whilst the relation to the other user focuses exclusively on how a person posting online information of all kinds follows that piece of information and reacts to other posters linking to that information.

Combining these two criteria together brings forth two major kinds of homo digitalis identities in term of the visibility of its presence on line: the stealthy and the open ones.

This stealthy feature does not relate to matters of anonymity the digital environment analysis may foster, nor questions of avatars and misleading or camouflaged identity the users may take when going online. This does not refer either to the idea of traceability which the core of other types of analysis. The stealthy homo digitalis simply represents the type of individual who, irrespective of age, gender or social class, uses the internet content just a source of information. $\mathrm{He}$ never uploads, he is the type of the downloader which is in the form of the retriever of information either literally saving web information on his local drive or reading 
or watching that information online. This type of homo digitalis fits best the category of the consumer who puts no effort into generating content but only in getting it.

The stealthy homo digitalis is on the other hand no different from the previous media consumer for which the communication process has always been one-sided. Pretty much the communication process the stealthy domo digitalis is involved in resembles that of a monologue where only just one of the part encodes information, while the second only decodes it. Stemming from this particular monological feature of the communication process the stealthy homo digitalis enact exclusively the role of audience.

But such role of audience is what, in its turn, divides the rather large class of stealthy homo digitalis in two smaller classes which we might name the gatherers and the hunters, categories which migrate easily depending on the interest on a certain topic. There is for the purpose of this identification of patterns of consumption a preference for using this two roles separate and not in combined formula as the evolutionary anthropologists do. The reasons for dubbing these as individual, distinct types of identities is generated by the way such digital individuals involve in the process of accessing information.

The gatherer stealthy homo digitalis is typically the subscriber to news feeds of any type, chat rooms, message boards, mailing lists or similar. He is in part the individual identified by Kosinetz as 'the lurker' [43] whose only interaction with the Internet content is simply harvesting whatever information is there at his disposal. But unlike in Kozinets identification of this class, the gatherer hardly makes any effort to migrate to another category of users, being constantly satisfied with what the digital medium has to offer.

The moment a gatherer starts to put some effort into searching whatever interests him, he migrates from the easy posture of a simple recipient of data to the more challenging position of hunting for more. The hunter is the individual not satisfied with the selection made for him by others, but more the searcher, the hyperlink individual. That particular type of interaction identity can be understood as the position of the active information seeker who cross-checks what is available from the received feeds replacing the horizontal trajectory of a simple gatherer with an in depth, vertical, detailed undertaking in data mining.

If the gatherer simply turns on the alert on new topics the webpages host, the hunter actively involves in searching for these new emergent topics besides simply being the beneficiary.

Taking the discussion a steps further, the moment a user abandons the stealthy identity that particular user migrates from the role of an audience to the role of the performer, which, in turn is understood as contentgenerating. The open homo digitalis is noticeable in the sense that he produces content, and not in that of leaving trace of his observance. His presence, on the other hand, is observable not simply by hitting a like button on a social media page or reposting a tweet, he is active in the sense of commenting those. Unlike the previous type of identity, the open digital individual grows increasingly present by offering a sort of feedback to the information available to him.

But this is only one side of his visibility which employs the use of some ready-made tools which have the purpose of expressing some feeling related to the content or just acknowledging that the information was seen. More than this, the visible identity is synonym to generating content. This feature stresses the two-dimensional character of the communication flow and involves the dialogical nature of the relation with the informational content of the Internet.

Having such characteristics in mind, the visible digital individual is not simply a consumer of the available information, but more, he becomes involved a process of manufacturing demand. Such visible identity for instance a blogger has leads to bouncing off ideas which is, above all, an offer made to an alleged general or niched audience. And the greater the impact such information has, 
the more evident the nature of manufactured demand is. And even more, in some case, like those in which the visible digital individual doubles his roles of a performer with that of following the reverberations such posts have converts him to a monitor position. This position, in turn, may be at least divided in two. On one hand, the monitor may simply act as a part in the dialogue an earlier post has generated or, on the other hand, may become a sort of truth holder position in the sense of censoring the ideas which he dislikes. The monitoring position converts in this particular instance in the role of an information dictatorship either for own-generated content or for others' when the open individual enacts the role of an administrator.

Furthermore, such open individuals may have various identities with various degrees of transparency either if it were to weigh it against the real life identity or against a constant avatar the individual may have online. But this last feature may have little if none impact on the user generated content since the discussion here does not aim to build correlations between the real and the digital identities, nor at the gap between the online and the offline ones.

Last but not least, these three types of instances the homo digitalis may consist of benefit from a very high level of fluidity in the sense of abandoning one and taking another of these identity as it is the users choices. More precisely, for instance, a stealthy homo digitalis may very easily migrate to ad hoc visible one as the web content may function as a trigger for such identity development and very easily migrating back once the purpose of such undertaking was reached.

Finally, another distinction is to be made here, a distinction that has been earlier anticipated. If the stealthy homo digitalis represents the relation to the poetic of the Internet content, the open homo digitalis is the picture of the relation to the poietic of the Internet.

\section{Conclusion}

As the discourse on human-computer interaction and computer-mediated communication shows both the methods and the scientific jargon are borrowed from neighbouring science. At the same time, the constant and rapid changed this field is subject to poses new difficulties with every new feature the softwared devices have. This is the reason why, to a much greater extent than any other social science the realities behind the concepts are constantly adapting. Cyber, virtual, digital, online, Internet based and so one proved thus to be challenging concepts which double the complexity of the medium the research is carried out on. These concepts that do not exclude themselves and trying to define very accurately each of the may become a rather far-fetched undertaking. They are as fluid as the realities they define and that is why a conceptual description can only be done in terms of maybe and possibly. The introduction of the homo digitalis identity makes no exception. It is only a class with various way of approximation based exclusively on the present stage of development of the softwared devices incorporating as seen various aspects the earlier described realities.

\section{Acknowledgement}

This paper is supported by the Sectorial Operational Programme Human Resources Development (SOP HRD), financed from the European Social Fund and by the Romanian Government under the contract number SOP HRD/159/1.5/S/136077

At the same, I would like to thank Acad. Florin Filip for being overly patient with reading my materials, discussing and supporting me during the entire duration of my postdoctoral research.

\section{References}

[1] C. Geertz, The Interpretation of Culture. New York: Basic Books, 2000 [1973], pp. 3-5

[2] A. Barnard and J. Spencer (eds.), The Routledge Encyclopedia of Social and 
Cultural Anthropology. London, New York: Routledge, 2012, pp. 368-369

[3] W. A. Darity Jr. (ed.), International Encyclopedia of Social Science. $2^{\text {nd }}$ Edition, Vol. 3, Betroit: MacMillan Reference USA, 2008, pp. 551-556

[4] W. A. Darity Jr. (ed.), International Encyclopedia of Social Science. $2^{\text {nd }}$ Edition, Vol. 3, Betroit: MacMillan Reference USA, 2008, p. 555

[5] R. Brunbaker and F. Cooper, "Beyond "identity", Theory and Society, vol. 29(1), p. 6, 2000

[6] W. A. Darity Jr. (ed.), International Encyclopedia of Social Science. $2^{\text {nd }}$ Edition, Vol. 3, Betroit: MacMillan Reference USA, 2008, p. 552

[7] E. Goffman, The Presentation of Self in Everyday Life, New York: Anchor Books, (? [1956]), p. 22

[8] J. Butler, Gender Trouble: Feminism and the Subversion of Identity, New York and London: Routledge, 2008 [1990], p. 59

[9] S. Turkle, The Second Self: Computers and the Human Spirit. Cambridge Massachusetts, London England The MIT Press, 2005 (1984)

[10] M. O'Neil, Cyberchiefs: Autonomy and Authority in Online Tribes. London, Pluto Press, 2009, p. 1

[11] M. O' Neil Cyberchiefs: Autonomy and Authority in Online Tribes London, Pluto Press, 2009, p. 1

[12] R. E. Hallet and K. Barber, 'Ethnographic Research in a Cyber Era' Journal of Contemporary Ethnography, vol. 43(3), pp. 306-330

[13] B. Danet, Cyberpl@y: Communicating online, Oxford, New York: Berg, 2001

[14] A. Marciano, "Living the VirtuReal: Negitiating Transgender Identity in Cyberspace", Journal of ComputerMediated Communication, Vol. 19(2014), p. $824-825$

[15] D. Hakken, Cyborgs@Cyberspace?: An Ethnographer Looks to the Future. New York, Routledge, 1999, p. 5
[16] W. A. Darity Jr. (ed.), International Encyclopedia of Social Science. $2^{\text {nd }}$ Edition, Vol. 2, Betroit: MacMillan Reference USA, 2008, p. 216

[17] M. Dodge and Rob Kitchin, Mapping Cuberspace, London and New York: Routledge, 2001, p. 1

[18] L. Strate, 'The Varieties of Cyberspace: Problems in Defining and Delimitations', Western Journal of Communication, vol. 63(3), p. 382, 1999

[19] L. Strate, 'The Varieties of Cyberspace: Problems in Defining and Delimitations', Western Journal of Communication, vol. 63(3), p. 384, 1999

[20] L. Strate, 'The Varieties of Cyberspace: Problems in Defining and Delimitations', Western Journal of Communication, vol. 63(3), p. 385, 1999

[21] M. Prensky, 'Digital Natives, Digital Immigrants Part 1', On the Horizon, vol. 9 (5), 2001, p. 1

[22] S. Turkle, The Second Self..., p. 32-64

[23] S. Turkle, The Second Self..., p. 155

[24] S. Turkle, The Second Self..., p. 247

[25] D. Hakken, Cyborgs@Cyberspace..., ibidem.

[26] D. Hakken, The Knowledge Landscapes of Cybespace, New York \& London: Routledge, 2003, p. 92

[27] C. Hine, Virtual Ethnography, London, Thousand Oaks, New Delhi, Sage Publications, 2000, p. 84

[28] C. Hine, Virtual Ethnography, London, Thousand Oaks, New Delhi, Sage Publications, 2000, p. 92

[29] M. O’Neil, Cyberchiefs..., p. 3

[30] H. Rheingold, Virtual Reality, New York: Summit Books, 1991, p. 122

[31] U. Eco, Six Walks in the fictional Woods, Harvard University Press, 1994

[32] T. Boellstorff, Coming of age in Second Life. An Anthropologist explores the Virtually Human, Princeton and Oxford: Princeton University Press, 2008, p. 157

[33] T. Boellstorff, Coming of age in Second Life. An Anthropologist explores the Virtually Human, Princeton and 
Oxford: Princeton University Press, 2008, p. 238

[34] T. Boellstorff, Coming of age in Second Life. An Anthropologist explores the Virtually Human, Princeton and Oxford: Princeton University Press, 2008, p. 238

[35] V. I. Propp, Morphology of the Folktale, Austin: University of Texas Press, 2009 [1928]

[36] T. Boellstorff, Bonie Nardi et al., Ethnography and the Virtual Worlds: A Handbook of Method, Princeton: Princeton University Press, 2013, p. 1

[37] T. Boelstorff, 'Rethinking Digital Anthropology' in Daniel Miller and Heather A. Horst, Digital Anthropology, London: Berg, 2012, p. 39

[38] T. Boelstorff, 'Rethinking Digital Anthropology' in Daniel Miller and Heather A. Horst, Digital Anthropology, London: Berg, 2012, p. 54

[39] R. Rogers, The End of the Virtual Digital Methods, text prepared for the Inaugural Speech, Chair, New Media \&
Digital Culture, University of Amsterdam, 8 May 2008, available online at http://www.govcom.org/rogers_oratie.pdf , retrieved July 20, 2015, 8:45

[40] R. V. Kizinets, Netnography: Doing Ethnographic Research Online, Los Angeles, London, New Delhi, Singapore, Washington DC: Sage, 2010, p. 33

[41] R. V. Kizinets, Netnography: Doing Ethnographic Research Online, Los Angeles, London, New Delhi, Singapore, Washington DC: Sage, 2010, p. 35

[42] J. Jouhki, The Anthropology of Homo Digitalis and His Tribes, 2009, available online at: http://techantropology.blogspot.ro/2009/1 1/anthropology-of-homo-digitalis-andhis.html and retrieved on July 21, 2015, 14.30

[43] R. V. Kozinets, Netnography: Doing Ethnographic Research Online, Los Angeles, London, New Delhi, Singapore, Washington DC: Sage, 2010, p. 34

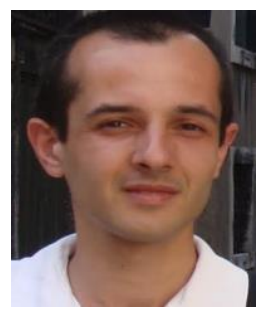

Adrian STOICESCU is a lecturer at the Department of Cultural Studies, Faculty of Letters, University of Bucharest. He has graduated philology in 2003, has an M.A. in Ethnology and Cultural Anthropology and also has a $\mathrm{PhD}$ in philology all from the University of Bucharest. His fields of interest are the internet studies, cultural anthropology, gender studies, and cultural studies approach to fictional texts. 